\title{
Age-related differences in function and structure of rat livers subjected to ischemia/reperfusion
}

\author{
Małgorzata Trocha ${ }^{1}$, Anna Merwid-Ląd ${ }^{1}$, Małgorzata Pieśniewska ${ }^{1}$, Joanna Kwiatkowska ${ }^{1}$, \\ Lidia Fereniec-Gołębiewska ${ }^{1}$, Przemysław Kowalski ${ }^{2}$, Adam Szeląg ${ }^{1}$, Tomasz Sozański ${ }^{1}$
}

\begin{abstract}
${ }^{1}$ Department of Pharmacology, Wroclaw Medical University, Wroclaw, Poland ${ }^{2}$ Department of Pathomorphology and Oncological Cytology, Wroclaw Medical University, Wroclaw, Poland
\end{abstract}

Submitted: 20 July 2015

Accepted: 17 December 2015

Arch Med Sci 2018; 14, 2: 388-395

DOI: https://doi.org/10.5114/aoms.2018.73470

Copyright $\odot 2018$ Termedia \& Banach

\section{Abstract}

Introduction: Liver function is affected during ischemia/reperfusion (IR). The current state of knowledge about liver aging processes during IR is incomplete. We evaluated the effects of aging on liver structure and function under IR conditions.

Material and methods: Animals were divided into control (C-2) and ischemia/reperfusion (IR-2) groups of young rats (2-4 months old) and C-12 and IR-12 groups of old rats (12-14 months old). The livers from IR-2 and IR-12 groups were subjected to partial ischemia $(60 \mathrm{~min})$, followed by global reperfusion (4 h). Blood samples were obtained during reperfusion (0, 30 and 240 $\mathrm{min}$ ) to estimate the activity of aminotransferases (ALT, AST). After IR, tumor necrosis factor- $\alpha$ (TNF- $\alpha$ ), interleukin-1b (IL-1b), malondialdehyde (MDA), and superoxide dismutase (SOD) were determined in liver homogenates.

Results: At all points of reperfusion, an increase in aminotransferase activity levels in the ischemic groups was observed; mainly between IR-12 and C-12 rats. The concentration of TNF- $\alpha$ was significantly higher in young animals (in non-ischemic groups: $p=0.09$, in ischemic groups: $p=0.05$ ). Under IR conditions, the concentration of IL-1b dropped $(p=0.05)$. The concentration of MDA was significantly higher in mature animals (in non-ischemic groups: $p=0.09$, in ischemic groups: $p=0.05$ ). In ischemic groups an increase in necrosis rate was observed regardless of age. Rats in the IR-12 group showed the most pronounced changes in hepatic architecture, including increased micro- and macrosteatosis and parenchymal cell destruction.

Conclusions: The function and structure of mature livers slightly deteriorate with age and these differences are more noticeable under IR conditions.

Key words: ageing, ischemia/reperfusion, liver, rat, oxidative stress, inflammation.

\section{Introduction}

Ischemia/reperfusion (IR) is considered to be the main cause of organ injury during such procedures as liver transplantation or hepatectomy [1]. This damage is initially caused by ischemia, and further aggravated by reperfusion. The reperfusion stage can be divided into the acute phase (3-6 h), involving generation of reactive oxygen species (ROS) and nitric oxide (NO) and activation of T-cells and Kupffer cells (KCs), and the subacute phase (18-24 h), characterized by neutrophil infiltration leading to continuous oxidant, cytokine, and chemokine production [2, 3].

\author{
Corresponding author: \\ Tomasz Sozański MD, PhD \\ Department \\ of Pharmacology \\ Wroclaw Medical \\ University \\ 2 Mikulicza-Radeckiego St \\ 50-345 Wrocław, Poland \\ Phone: +48717841440 \\ Fax: +48717840094 \\ E-mail: tsoz@wp.pl
}


During IR-evoked oxidative stress, generation of ROS may exceed the capacity of antioxidative systems. Malondialdehyde (MDA) is the most commonly studied product of polyunsaturated fatty acid peroxidation, and it is widely used as an indicator of oxidative damage. Superoxide dismutases (SODs) are antioxidant enzymes that catalyze the dismutation of superoxide $\left(\mathrm{O}_{2}{ }^{--}\right)$into oxygen or hydrogen peroxide [2].

Tumor necrosis factor- $\alpha$ (TNF- $\alpha$ ), released from $\mathrm{KCs}$ in the initial phase of reperfusion, intensifies the expression of adhesion molecules and release of other cytokines. These changes are responsible for neutrophil activation and their accumulation in the later phase $[4,5]$. Interleukin-1 $\beta$ (IL-1 $\beta)$ is a proinflammatory cytokine released from KCs in response to TNF- $\alpha$. Similarly to TNF- $\alpha$, IL-1 $\beta$ can up-regulate adhesion proteins on neutrophils and induce IL- 8 synthesis. It can also interact with neutrophils and stimulate the release of ROS. Another property of this molecule is the augmentation of TNF- $\alpha$ synthesis by KCs [5].

The liver function appears to be well maintained in old age [6]. However, the risk of failure of the mature liver after IR appears to be increased [7]. Increased morbidity and mortality after vascular clamping [8, 9], as well as poor long-term survival after transplantation [10], have been observed. Although in some papers age-related differences in the liver response to IR have been reported [11-13], our knowledge is still incomplete.

The aim of the study was to evaluate age-related differences in function and structure of rat livers subjected to IR and to investigate how aging influences selected parameters of inflammation and oxidative stress under both physiological and IR conditions.

\section{Material and methods}

\section{Animals}

The study was carried out on Wistar male rats obtained from the Animal Laboratory of the Department of Pathological Anatomy, Wroclaw Medical University. Before the experiment, the animals had been housed in standard conditions (12: 12 day/night cycle, stable temperature $19-21^{\circ} \mathrm{C}$, humidity $45-60 \%$, and continuous ventilation) and had ad libitum access to food and water. The experiment was performed in accordance with the NIH Guide for the Care and Use of Laboratory Animals and was approved by the First Local Ethics Committee on Animal Research of the Institute of Immunology and Experimental Therapy, Polish Academy of Sciences in Wroclaw.

\section{Experimental protocol}

After adaptation, the animals were divided into two groups (C-2 and IR-2) of young rats ( $2-4$ months old) and two groups (C-12 and IR-12) of old rats (1214 months old). Rats belonging to the C-2 ( $n=10)$ and the C-12 $(n=9)$ groups were not subjected to $\mathrm{IR}$, and rats from the IR-2 $(n=9)$ and the IR-12 $(n=$ 9) groups were subjected to 60 min of partial ischemia followed by $4 \mathrm{~h}$ of global reperfusion.

\section{Preparation of the liver IR injury model}

The rats were weighed and anesthetized with intramuscular injection of ketamine hydrochloride $(7 \mathrm{mg} / \mathrm{kg}$ ) (Bioketan, Vetoquinol Biowet, Poland), medetomidine hydrochloride $(0.1 \mathrm{mg} / \mathrm{kg})$ (Domitor, amp. $1 \mathrm{mg} / \mathrm{ml}$, Orion Pharma, Finland), and butorphanol tartrate $(2 \mathrm{mg} / \mathrm{kg}$ ) (Morphasol, amp. $4 \mathrm{mg} / \mathrm{ml}$, aniMedica GmbH Germany). After a suitable level of anesthesia was achieved, rats from IR-2 and IR-12 groups underwent midline laparotomy. Seventy percent liver ischemia (left lateral and median lobes) was achieved by occluding the branches of the portal vein and hepatic artery using a microvascular clip. The rats were given heparin $(200 \mathrm{U} / \mathrm{kg}$ ) (Heparinum WZF - amp. $25000 \mathrm{U} / 5 \mathrm{ml}$, Polfa Warszawa, Poland), to prevent blood coagulation. After $60 \mathrm{~min}$ of ischemia, the clip was removed to allow reperfusion for $4 \mathrm{~h}$. The abdomen was closed and the rats were observed during reperfusion. During whole reperfusion rats remained asleep. In the rats from C- 2 and $\mathrm{C}-12$ groups, the branches of the portal vein and the hepatic artery were isolated, but not occluded. Blood samples $(0.8 \mathrm{ml})$ were obtained after catheterization of the tail vein, just before ischemia and after 30 and 240 min of reperfusion to determine the levels of alanine and asparagine aminotransferases (ALT, AST). All blood samples were replaced by the same volume of saline solution (sol. $0.9 \%$ sodium chloride (Polpharma S.A., Poland)). When the experiment was terminated, the livers were weighted and ischemic lobes were isolated.

After the reperfusion, one batch of ischemic hepatic tissues was fixed in $0.1 \mathrm{M}$ phosphate buffer with $2 \%$ paraformaldehyde and $2 \%$ glutaraldehyde ( $\mathrm{pH} 7.6)$, and the other batch of the ischemic tissues was homogenized on ice with the lysis buffer (140 mM NaCl, 10 mM EDTA, 10\% glycerol, $1 \%$ NP40, $20 \mathrm{mM}$ Tris base, $\mathrm{pH}$ 7.5). The homogenized tissues were subsequently centrifuged at $14000 \mathrm{rpm}$ for $25 \mathrm{~min}$, at $4^{\circ} \mathrm{C}$ and the resulting supernatants were collected [14].

\section{Parameters of oxidative stress and inflammation}

Malondialdehyde and SOD were estimated in the liver homogenates using colorimetric methods (spectrophotometer MARCEL S350 PRO). SOD activity was assayed using a RANSOD kit (Randox Laboratories, Crumlin, UK). Malondialde- 
hyde concentration was determined with BIOXYTECH-MDA-586 (OxisResearch, USA), according to the manufacturer's instructions. TNF- $\alpha$ and IL- $1 \beta$ plasma levels were determined using commercially available Rat TNF- $\alpha$ ELISA and Rat IL-1 $\beta$ ELISA kits (Diaclone SAS, France).

\section{Biochemical analyses}

Serum activity of ALT and AST and protein concentration in the homogenates were assayed using commercial enzymatic methods in a certified laboratory.

\section{Histological examination}

Different regions of the ischemic and non-ischemic livers were fixed in $10 \%$ formalin and embedded in paraffin. Sections of $4.5 \mu \mathrm{m}$ were made and stained with hematoxylin-eosin. Then, they were histologically evaluated under a light microscope for the severity of ischemic necrosis, degree of steatosis as percentage of the microscopic field (small cytoplasmic vacuoles containing lipids or single fat droplets displacing the nuclei of hepatocytes), neutrophil infiltration and destruction of hepatic architecture [15]. The necrosis rate was semi-quantitatively determined as follows: absent -0 ; 0-25\% necrosis per microscopic field -1 ; $25-$ $50 \%$ necrosis per microscopic field - 2; 50-75\% necrosis per microscopic field - 3; 75-100\% necrosis per microscopic field -4 .

\section{Statistical analysis}

Data were expressed as mean values \pm SD. Statistical analysis of the age and IR effects on the oxidative stress and inflammatory parameters was performed using a two-way analysis of variance

A

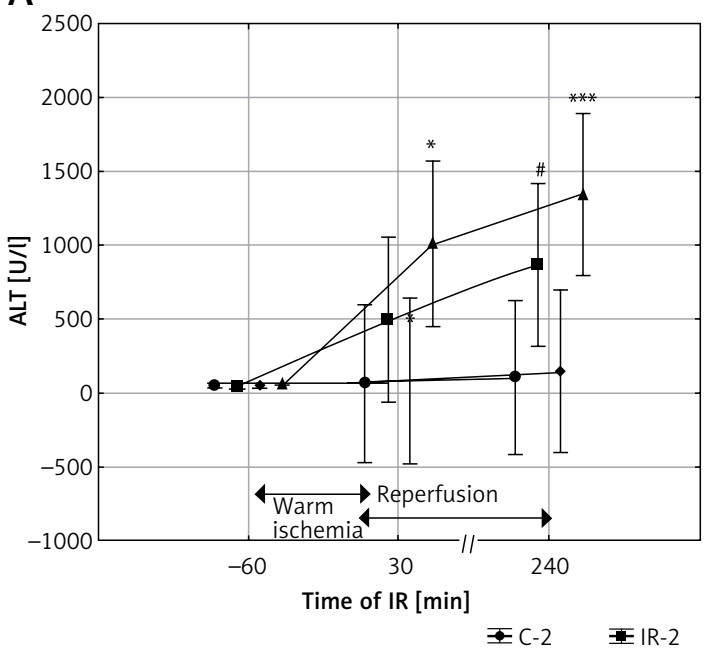

(ANOVA). Statistical analysis of the effect of rat age and time of reperfusion on ALT and AST levels was performed using MANOVA with repetitions. Contrast analysis was used for specific comparisons. Hypotheses were considered positively verified if $p<0.05$.

\section{Results}

\section{ALT and AST activity}

Alanine aminotransferase activity measured before IR was comparable in all groups of rats regardless of age, but AST activity was the highest in the IR-12 group (IR-12 vs. C-2 and IR-2, $p<0.001$, and IR-12 vs. C-12, $p<0.05)$. During reperfusion, an increase in both ALT and AST activity in the ischemic groups was observed. After $30 \mathrm{~min}$ of reperfusion, the activity of both aminotransferases was significantly higher in the IR-12 than in the C-12 group (IR-12 vs. C-12, $p<0.05$, for both enzymes). After $240 \mathrm{~min}$ of reperfusion, the activity of both aminotransferases was also significantly different in mature groups (IR-12 vs. C-12, $p<0.05$ for AST and IR-12 vS. C-12, $p<0.005$ for ALT). At this point in time the increase in ALT activity was also significant in the younger group that underwent IR (IR-2 vs. C-2, $p<0.05)$ and the increase in AST activity was bordering on significance (IR-2 vS. $\mathrm{C}-2, p=0.08$ ) (Figures $1 \mathrm{~A}, \mathrm{~B}$ ).

\section{Cytokine levels}

In the ischemic groups the concentration of TNF- $\alpha$ was slightly higher than in the corresponding non-ischemic groups. In both non-ischemic and ischemic groups, the concentration of TNF- $\alpha$ was significantly higher in young animals than in the older ones (C-12 vs. C-2, $p<0.01$, and IR-12 vs.

\section{B}

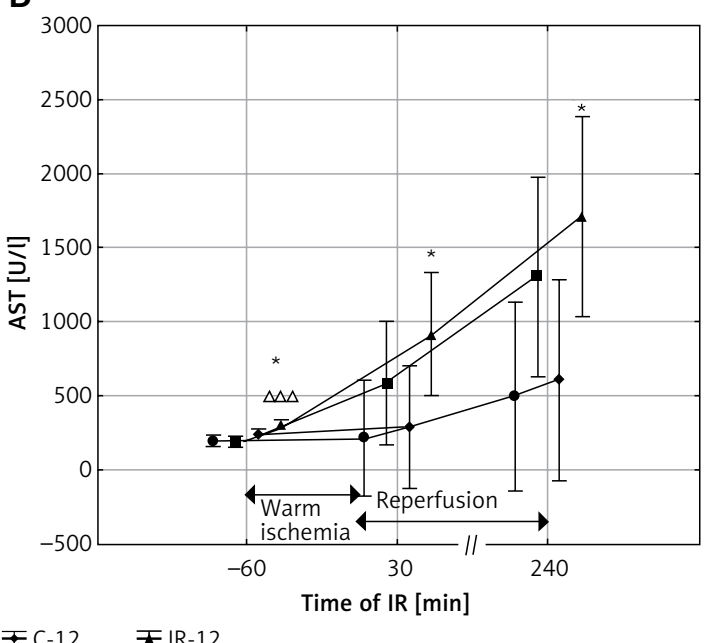

Figure 1. The effect of IR and aging on ALT (A) and AST (B) activity

The values are presented as mean $\pm S D$. C-2 -young rats non-subjected to IR, C-12-mature rats not subjected to IR, IR-2-young rats subjected to $I R, I R-12$ - mature rats subjected to $I R ;{ }^{*} p<0.05$ and ${ }^{* * *} p<0.005$ (IR-12 vs. C-12), ${ }^{*} p<0.05$ (IR-2 vs. C-2), ${ }^{\Delta \Delta \Delta} p<0.005$ (IR-12 vs. IR-2). 
A

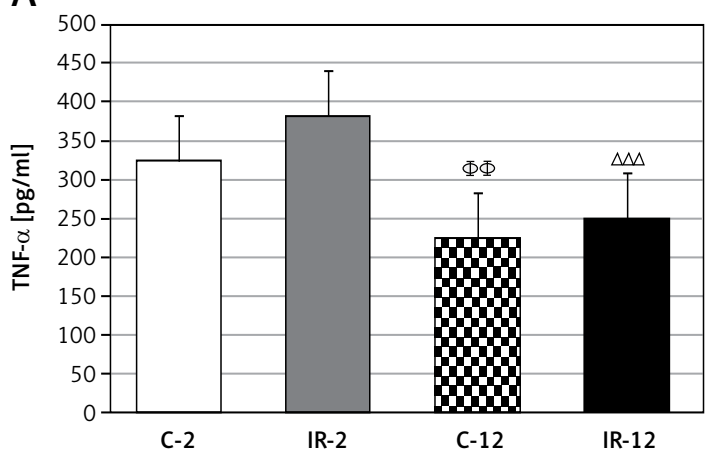

B

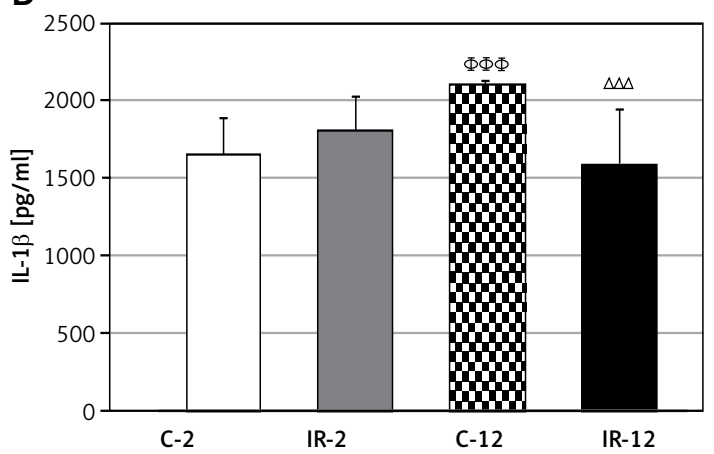

Figure 2. The effect of IR and aging on cytokine (TNF- $\alpha(\mathbf{A})$ and IL-1 $\beta(B))$ levels

The values are presented as mean $\pm S D$. C-2 -young rats non-subjected to IR, $C$-12-mature rats not-subjected to IR, IR-2-young rats subjected to IR, IR-12 - mature rats subjected to IR; ${ }^{\Delta \Delta \Delta} p<0.005$ (IR-12 vs. IR-2), ${ }^{\Phi \Phi} p<0.01$ and ${ }^{\Phi \Phi \Phi} p<0.005$ (C-12 vs. C-2).

IR-2, $p<0.005)$ (Figure $2 \mathrm{~A}$ ). Concentration of IL-1 $\beta$ in the non-ischemic groups depended on age and it was significantly higher in the mature group (C-12 vs. C-2, $p<0.005$ ). Under IR conditions, the concentration of IL-1 $\beta$ dropped in the IR-12 group (IR-12 vs. C-12, $p<0.005$ ) and increased in the IR-2 group, which is why the difference between young and mature ischemic rats was on the border of significance (IR-12 vs. IR-2, $p=0.053$ ) (Figure $2 \mathrm{~B}$ ).

\section{Parameters of oxidative stress}

Under IR conditions, the changes in SOD activity were not significant, but were slightly less pronounced than in the corresponding non-ischemic age groups (Figure $3 \mathrm{~A}$ ). The concentration of MDA depended on age and increased significantly in both ischemic and non-ischemic mature animals (C-12 vs. C-2, and IR-12 vs. IR-2, $p<0.01$, in both variants). Only a negligible increase in MDA under IR conditions was observed (Figure $3 \mathrm{~B}$ ).

\section{Histological findings}

No significant differences in the hepatic structure were seen in either young or mature non-isch- emic animals (C-2, C-12). Livers from those groups featured normal architecture and only a slight degree of steatosis. In the ischemic groups (IR-2, IR-12), an increase in the necrosis rate associated with intense neutrophil recruitment was observed regardless of age (Figure 4). Given the necrosis score value (NSV), the difference between young ischemic and non-ischemic rats was bordering on significance (IR-2 vs. C-2, $p=0.06$ ). More pronounced differences were observed between the mature groups (IR-12 vs. C-12, $p<0.05$ ). Adult ischemic rats (IR-12) showed the most noticeable changes in the hepatic architecture, including increased micro- and macrosteatosis and parenchymal cell destruction. Also the percentage of steatosis was the highest in this group (IR-12 vs. C-2 and C-12, $p<0.01$, and IR-12 vs. IR-2, $p<0.05$ ) (Table I).

\section{Discussion}

In this study we focused on the age-dependent changes in the liver function and structure, as well as oxidative stress and proinflammatory parameters during IR. We observed that (1) the increase in ALT and AST activity was significantly higher in mature than in young animals, especially in the
A

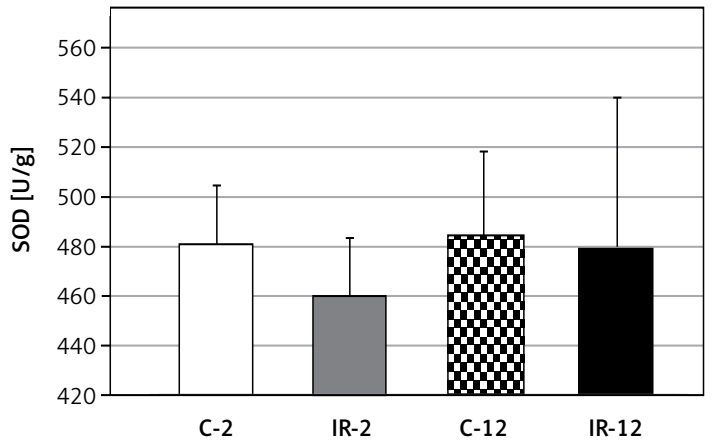

B

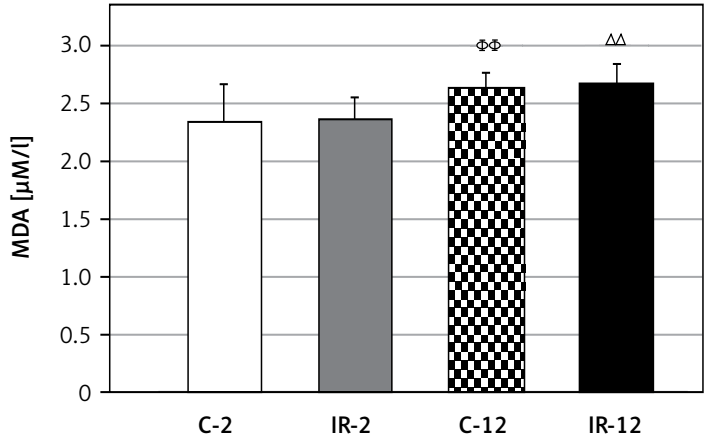

Figure 3. The effect of IR and aging on the activity of SOD (A) and the concentration of MDA (B)

The values are presented as mean $\pm S D$. C-2 -young rats non-subjected to IR, C-12-mature rats not-subjected to IR, IR-2-young rats subjected to IR, IR-12 - mature rats subjected to IR; ${ }^{\Delta \Delta} p<0.01$ (IR-12 vs. IR-2), ${ }^{\Phi \Phi} p<0.01$ (C-12 vs. C-2). 

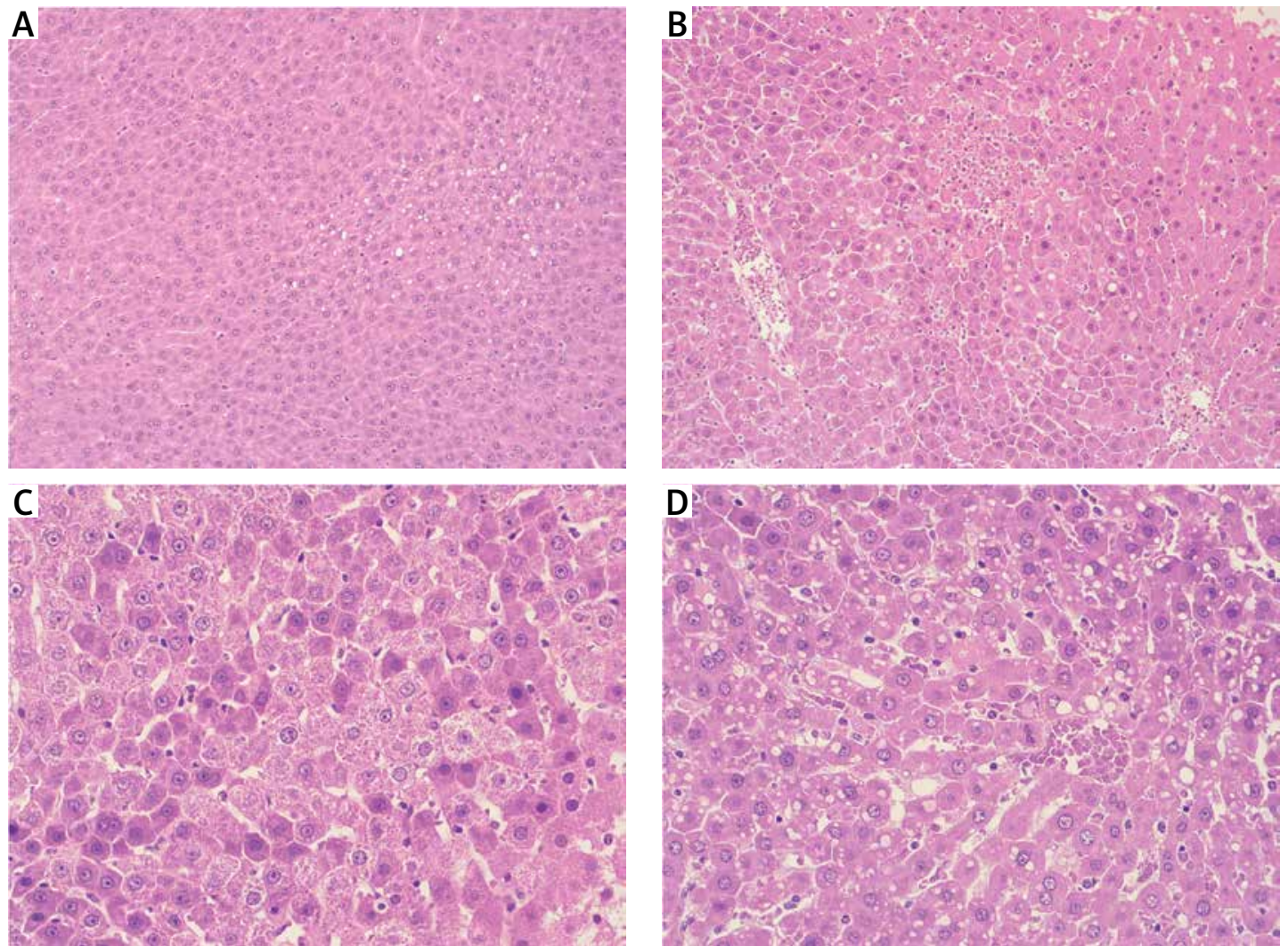

Figure 4. Histopathological examination of liver tissue (stained with hematoxylin-eosin) from group C-2 - young rats non-subjected to IR (A), group IR-2 - young rats subjected to IR (B), group C-12 - mature rats not subjected to IR (C), and from group IR-12 - mature rats subjected to IR (D). Livers from C-2 and C-12 groups featured normal architecture and only low degree of steatosis, but from IR-2 and IR-12 groups presented higher rate of the necrosis associated with intense neutrophil infiltrate

groups that underwent IR; (2) enzymatic changes were corroborated by histological findings, as a significant increase in necrosis rate in both ischemic groups, and severe micro- and macrosteatosis and parenchymal cell destruction in mature ischemic rats were observed; (3) changes in the proinflammatory parameters indicated the differentiated age-dependent reaction in IR conditions: the level of TNF- $\alpha$ was significantly higher in young rats, independent from IR, whereas the level of IL-1 $\beta$ was higher in old animals, but decreased under IR; (4) in mature rats oxidative stress was more pronounced as confirmed by significantly higher MDA concentration.

In non-ischemic groups, AST activity was significantly higher in mature than in young rats. Therefore, despite a lack of histological abnormalities, we may suspect a low-degree, age-related liver dysfunction in old animals. It is well known that liver function deteriorates during IR. As we found in a previous study [16], the most pronounced liver injury was detected in the groups exposed to IR - the activity of ALT and AST increased during hepatocyte damage. Similarly to other research

Table I. The necrosis score value (NSV) and the percentage of steatosis in microscopic fields in all examined groups

\begin{tabular}{|lcccc|}
\hline Variable & \multicolumn{2}{c|}{ Steatosis (\% of microscopic field) } & \multicolumn{2}{c|}{ Necrosis (NSV) } \\
\cline { 2 - 5 } & Mean & SD & Mean & SD \\
\hline C-2 & 0.30 & 0.42 & 0.10 & 0.32 \\
\hline IR-2 & $2.44^{\#}$ & 3.64 & $0.67^{\#}$ & 0.87 \\
\hline C-12 & $0.22^{\# \#}$ & 0.36 & 0.11 & 0.33 \\
\hline IR-12 & $11.56^{* * \Delta}$ & 16.48 & $0.78^{*}$ & 0.83 \\
\hline
\end{tabular}

The values are presented as mean $\pm S D$. C-2 - young rats non-subjected to $I R, C$ - 12 - mature rats not-subjected to IR, IR-2 - young rats subjected to IR, IR-12 - mature rats subjected to IR; the difference in steatosis: ${ }^{\star *} p<0.01$ (IR-12 vs. C-12), ${ }^{\Delta} p<0.05$ (IR-12 vs. IR-2); the difference in necrosis: ${ }^{*} p=0.06$ (IR-2 vs. C-2), ${ }^{*} p<0.05$ (IR-12 vs. C-12). 
[17], we also observed age-related susceptibility to IR. Differences in the enzymatic activities between the IR-exposed and non-exposed livers appeared at the time points of 30 and 240 min of reperfusion in the mature animals. In young animals, significant differences appeared only at $240 \mathrm{~min}$ of reperfusion. Hence, the conclusion is that the livers obtained from young animals are less susceptible to injury, than the livers harvested from mature rats. These findings were supported by histological analyses. The histological evaluation of liver specimens revealed necrotic foci with inflammatory infiltrates of similar magnitude, regardless of age. Age differences were associated with the degree of steatosis. Similarly to the study of Kireev et al. [18], the degree of steatosis after IR was greater in the group of mature animals. Our results are also consistent with those obtained by Okaya et al. [19]; however, these authors also revealed significant differences in leukocyte infiltration. In our study, leukocyte infiltration in the specimens was comparable in all ischemic groups. Steatosis is one of the determinants of reversible cell injury; therefore its significantly higher degree in ischemic organs derived from old rats, together with increased aminotransferase activity, indicates age-related injury.

It was observed that neutrophil accumulation in the lobes with sustained blood flow was the highest after 6 h of reperfusion [20], and their number correlated well with intensified ROS production in reperfused tissues [21]. Neutrophils adhere to the endothelium and pass into liver parenchyma, causing hepatocyte injury due to cell granule degradation and release of ROS and proteolytic enzymes, e.g. proinflammatory cytokines such as TNF- $\alpha$ [21, 22]. In our study, the time of reperfusion was shorter ( $4 \mathrm{~h}$ ) and neutrophils accumulated in the ischemic tissue in similar amounts, regardless of age. Therefore, age-related differences in MDA and TNF- $\alpha$ concentrations suggest KCs as a probable source of ROS and proinflammatory cytokines [23]. KCs were shown to be activated during the first $6 \mathrm{~h}$ of reperfusion [24], and their activation in livers derived from older rats was impaired [25].

At the end of the experiment the level of TNF- $\alpha$, was determined in liver homogenates. Its action in an ischemic liver is multidirectional. TNF- $\alpha$ influences, among other things, cell proliferation and apoptosis, proinflammatory cytokines and ROS release, and enhances neutrophil adhesion and blood coagulation [5]. In contrast to some other studies [12, 16, 26-28], in our study the level of TNF- $\alpha$ was only slightly increased in the livers subjected to IR. Detailed comparison of our results with the results obtained by other authors is difficult due to different duration of IR or different animal species. TNF- $\alpha$ is produced in extensive amounts during the sub-acute phase of reperfusion [29]. Our experiment was designed in such a way that the reperfusion phase lasted for only $4 \mathrm{~h}$ and probably was too short to show significant differences between the ischemic and non-ischemic groups. In our study, the level of TNF- $\alpha$ depended on age. Its values were significantly higher in the young than in the old rats. Okaya et al. also found that the production of this cytokine by KCs was greater in younger rats [19]. Kireev et al. described increased expression of mRNA for various proinflammatory cytokines under IR. In the case of TNF- $\alpha$ that increase was not as pronounced in the mature rats as in the younger ones [18]. IL-1 $\beta$ was another cytokine determined in our work in liver homogenates after the reperfusion period. The level of IL-1 $\beta$ was significantly higher in the mature animals than in the young ones. In response to $I R$, the level of this cytokine increased significantly in the younger livers, but decreased in the mature ones. Considering the extent of histological changes in the livers taken from old rats and the increase in aminotransferase activity in the same groups, it may be suspected that the lower proinflammatory cytokine level in these groups, as well as a lack of their increase under IR, may result from impaired $K C$ and hepatocyte function in old rats.

While analyzing MDA concentration in the examined groups, it may be noted that the severity of oxidative stress was age-dependent and it was greater in the mature rats than the young ones. In the group of young rats characteristic changes in the oxidative stress parameters, such as increased level of MDA and reduced SOD activity, were found after IR. The observed changes were not statistically significant. In various reports, however, SOD activity also remained unaffected, suggesting that this enzyme is not a sensitive predictor of oxidative stress [30, 31]. The insignificant increase in MDA [32, 33] could be a result of too short duration of ischemia and reperfusion. In those conditions, liver injury could not be sufficiently manifested. Probably MDA or SOD levels should also be measured after 24 to $48 \mathrm{~h}$, during the second phase of reperfusion. Another possibility of evoking more pronounced oxidative stress is development of another experimental model of global ischemia and reperfusion [34]. The lack of changes in MDA level in old livers in our experiment could be the result of an age-dependent impaired response of the organs to oxidative stress under IR conditions. Evaluation of post-ischemic ROS formation by chemiluminescent real-time imaging [35] indicated that age appears to be an important factor affecting liver sensitivity to oxidative stress. During reperfusion the livers of mature rats generated a lower amount of ROS, as compared to the livers obtained from young animals.

In conclusion, we conclude that the influence of IR on liver function and structure, as well as on 
oxidative stress and inflammation, depends on age. Based on both aminotransferases activity and histological findings, we found that hepatocyte damage was more pronounced in the mature livers than in the younger ones, especially under IR conditions. Age-related differences were also visible in the case of MDA and TNF- $\alpha$, but only a slight increase in the levels of those parameters was observed following IR. Therefore, further experiments are probably needed, using a longer reperfusion period or a global ischemia model. An interesting question is why the changes of prooxidative and proinflammatory parameters were weaker in the groups of mature animals. A few possible explanations of the lower TNF- $\alpha$ and MDA levels in the mature animals and the drop in IL-1 $\beta$ concentration in the mature animals in response to IR could be offered. Similarly to the earlier suggestion of Gasbarrini et al. [35] the reasons for our results could also be reduced activity of KCS (the source of ROS in the initial period of reperfusion), impaired function of mitochondria (the main intracellular sources of ROS), and reduced blood flow in mature livers. Therefore, in order to better understand these results, our data should be supplemented by an analysis of additional parameters, such as KC activation or liver blood flow. Hence, the results of this study may just indicate that changes in liver function and structure, as well as particular parameters determining the redox and inflammatory state, depend on age and may be the reason for variable liver responses to such pathological conditions as those observed under IR, but some of our findings should be confirmed in subsequent studies.

\section{Conflict of interest}

The authors declare no conflict of interest.

\section{References}

1. Cobreros A, Sainz L, Lasheras B, Cenarruzabeitia E. Hepatotoxicity of ethanol: protective effect of calcium channel blockers in isolated hepatocytes. Liver 1997 17: 76-82.

2. Fan C, Zwacka RM, Engelhardt JF. Therapeutic approaches for ischemia/reperfusion injury in the liver. J Mol Med 1999; 77: 577-96.

3. Hines IN, Harada H, Flores S, Gao B, McCord JM, Grisham MB. Endothelial nitric oxide synthase protects the post-ischemic liver: potential interactions with superoxide. Biomed Pharmacother 2005; 59: 183-9.

4. Peralta C, Fernández L, Panés J, et al. Preconditioning protects against systemic disorders associated with hepatic ischemia-reperfusion through blockade of tumor necrosis factor-induced P-selectin up-regulation in the rat. Hepatology 2001; 33: 100-13.

5. Perry BC, Soltys D, Toledo AH, Toledo-Pereyra LF. Tumor necrosis factor-alpha in liver ischemia/reperfusion injury. J Invest Surg 2011; 24: 178-88.

6. Fu A, Nair KS. Age effect on fibrinogen and albumin synthesis in humans. Am J Physiol 1998; 275: E1023-30.
7. Feng S, Goodrich NP, Bragg-Gresham JL, et al. Characteristics associated with liver graft failure: the concept of a donor risk index. Am J Transplant 2006; 6: 783-90.

8. Le Couteur DG, Rivory LP, Pond SM. The effects of aging and nutritional state on hypoxia-reoxygenation injury in the perfused rat liver. Transplantation 1994; 58: 531-6.

9. Clavien PA, Selzner M, Rudiger HA, et al. A prospective randomized study in 100 consecutive patients undergoing major liver resection with versus without ischemic preconditioning. Ann Surg 2003; 238: 843-50.

10. Collins BH, Pirsch JD, Becker YT, et al. Long-term results of liver transplantation in patients 60 years of age and older. Transplantation 2000; 70: 780-3.

11. Trocha M, Szelag A, Pieśniewska M, Fereniec-Gołębiewska L, Grotthus B, Merwid-Ląd A. Effect of aging process on liver function in extracorporeal rat liver perfusion. Hepatogastroenterology 2007; 54: 1207-11.

12. Abe Y, Hines IN, Zibari G, et al. Mouse model of liver ischemia and reperfusion injury: method to study reactive oxygen and nitrogen metabolites in vivo. Free Radic Biol Med 2009; 46: 1-7.

13. Kireev RA, Cuesta S, Ibarrola C, et al. Age-related differences in hepatic ischemia/reperfusion: gene activation, liver injury, and protective effect of melatonin. Surg Res 2012; 178: 922-34.

14. Morales Al, Vicente-Sanchez C, Jerkic M, Santiago JM, Sanchez-Gonzalez PD, Perez-Barriocanal F. Effect of quercetin on metallothionein, nitric oxide synthase and cyclooxygenase-2 expression on experimental chronic cadmium nephrotoxicity in rats. Toxicol Appl Pharmacol 2006; 210: 128-35.

15. Takeda Y, Arii S, Kaido T, et al. Morphologic alteration of hepatocytes and sinusoidal endothelial cells in rat fatty liver during cold preservation and the protective effect of hepatocyte growth factor. Transplantation 1999; 67: 820-8.

16. Trocha M, Merwid-Lą A, Chlebda E, et al. Influence of ezetimibe on selected parameters of oxidative stress in rat liver subjected to ischemia/reperfusion. Arch Med Sci 2014; 10: 817-24.

17. Selzner M, Selzner N, Chen L, et al. Exaggerated up-regulation of tumor necrosis factor alpha-dependent apoptosis in the older mouse liver following reperfusion injury: targeting liver protective strategies to patient age. Liver Transpl 2009; 15: 1594-604.

18. Kireev RA, Cuesta S, Ibarrola C, et al. Age-related differences in hepatic ischemia/reperfusion: gene activation, liver injury, and protective effect of melatonin. J Surg Res 2012; 178: 922-34.

19. Okaya T, Blanchard J, Schuster R, et al. Age-dependent responses to hepatic ischemia/reperfusion injury. Shock 2005; 24: 421-7.

20. Jaeschke H, Farhood A, Smith CW. Neutrophils contribute to ischemia-reperfusion injury in rat liver in vivo. FASEB 1990; 4: 3355-9.

21. Lentsch AB, Yoshidone $H$, Cheadle WG, Miller FN, Edwards MJ. Chemokine involvement in hepatic ischemia/ injury in mice: roles of macrophage inflammatory protein-2 and KC. Hepatology 1998; 27: 1172-7.

22. Lentsch $A B$, Kato $A$, Yoshidone $H$, McMastres KM, Edwards MJ. Inflammatory mechanisms and therapeutic strategies for warm hepatic ischemia/reperfusion injury. Hepatology 2000; 32: 169-73.

23. Grewe M, Gausling R, Gyufko K, Hoffmann R, Decker K. Regulation of the mRNA expression for tumor necrosis factor alpha in rat liver macrophages. J Hepatol 1994; 20: 811-8. 
24. Nakamitsu A, Hiyama E, Imamura Y, Matsuura Y, Yokoyama T. Kupffer cell function in ischemic and nonischemic livers after hepatic partial ischemia/reperfusion. Surg Today 2001; 31: 140-8.

25. Caldwell-Kenkel JC, Currin RT, Tanaka Y, Thurman RG, Lemasters JJ. Kupffer cell activation and endothelial cell damage after storage of rat livers: effect of reperfusion. Hepatology 1991; 13: 83-95.

26. Ma W, Wang ZR, Shi L, Yuan Y. Expression of macrophage inflammatory protein-1alpha in Kupffer cells following liver ischemia or reperfusion injury in rats. World J Gastroenterol 2006; 12: 3854-8.

27. Aldemir D, Tufan H, Tecder-Unal M, et al. Age-related alterations of oxidative stress and arginase activity as a response to intestinal ischemia-reperfusion in rat kidney and liver. Transplantation Proceedings 2003; 35: 2811-5.

28. Colletti LM, Remick DG, Burtch GD, Kunkel SL, Strieter RM, Campbell DA Jr. Role of tumor necrosis factor-alpha in the pathophysiologic alterations after hepatic ischemia/reperfusion injury in the rat. J Clin Invest 1990; 85: 1936-43.

29. Nakamitsu A, Hiyama E, Imamura Y, Matsuura Y, Yokoyama T. Kupffer cell function in ischemic and nonischemic livers after hepatic partial ischemia/reperfusion. Surg Today 2001; 31: 140-8.

30. Ceylan A, Karasu C, Aktan F, Güven C, Can B, Ozansoy G. Effects of simvastatin treatment on oxidant/antioxidant state and ultrastructure of diabetic rat myocardium. Gen Physiol Biophys 2003; 22: 535-47.

31. Ozansoy G, Akin B, Aktan F, Karasu Ç. Short-term gemfibrozil treatment reverses lipid profile and peroxidation but does not alter blood glucose and tissue antioxidant enzymes in chronically diabetic rats. Mol Cell Biochem 2001; 216: 59-63.

32. Xue F, Zhang JJ, Xu LM, Zhang C, Xia Q. Protective ef fects of HGF-MSP chimer (metron factor-1) on liver ischemia-reperfusion injury in rat model. J Dig Dis 2010; 11 : 299-305.

33. Wu C, Wang P, Rao J, et al. Triptolide alleviates hepatic ischemia/reperfusion injury by attenuating oxidative stress and inhibiting NF-kappaB activity in mice. J Surg Res 2011; 166: e205-13.

34. Spiegel HU, Bahde R. Experimental models of temporary normothermic liver ischemia. J Invest Surg 2006; 19: 113-23.

35. Gasbarrini A, Pasini P, Nardo B, et al. Chemiluminescent real time imaging of post-ischemic oxygen free radicals formation in livers isolated from young and old rats. Free Radic Biol Med 1998; 24: 211-6. 